\title{
Gabapentina preoperatoria como adyuvante en el manejo del dolor agudo postoperatorio en histerectomía abdominal
}

\author{
J. A. Batista ${ }^{1}$ y M. M. Errigo ${ }^{2}$ \\ ${ }^{1}$ Servicio de Anestesiología y ${ }^{2}$ Unidad de Clínica del Dolor. Departamento de Áreas Críticas. Hospital Santo \\ Tomás. Panamá
}

Batista JA, Errigo MM. Gabapentina preoperatoria como adyuvante en el manejo del dolor agudo postoperatorio en histerectomía abdominal. Rev Soc Esp Dolor 2015; 22(5):200-204.

\begin{abstract}
Background: It is well known that pain is subjective symptom and postoperative pain continues prevalence worldwide, because despite all the progress has not yet been able to eradicate completely.

Objective: To evaluate the adjuvant effect on postoperative pain intensity $600 \mathrm{mg}$ of gabapentin administered preoperatively in patients undergoing abdominal hysterectomy at St. Thomas Hospital from April to August 2014.

Methods: An experimental study cohort prospective randomized, controlled, double blind study in patients undergoing abdominal hysterectomy was performed under general anesthesia. Were randomized into two groups: gabapentin group -gabapentin $600 \mathrm{mg}$ (2 hours preoperative) + analgesia morphine through pump patient controlled analgesia (PCA)- and control group -analgesia with morphine via PCA pump; morphine dose was calculated based on weight ranges to $0.016 \mathrm{mg} / \mathrm{kg} / \mathrm{h}$. Pain intensity scale with verbal numeric scale (VNS) of 11 points at 2, 6 and 24 hours were evaluated, the number of rescues administered postoperatively was quantified and the prevalence of adverse events in both groups was measured.

Results: Seventy-two patients splitted into two homogeneous groups were recruited, the average age was $44.72 \pm$ 6.17 years in gabapentin group and $49.36 \pm 9.31$ years in control group. As for postoperative pain was statistically significant difference between the two groups ( $p<0.05)$, a difference was found in the VNS of 11 points, $4.27 \pm 2.32,1.94 \pm 1.95$ and
\end{abstract}

Recibido: $18-12-14$

Aceptado: 15-03-15
$0.77 \pm 1.28$ in gabapentin group and $7.13 \pm 2.41,4.5 \pm 2.37$ y $3.25 \pm 2.18$ in control group at 2, 6 and 24 hours respectively; the amount of ransom administered by PCA pump during the first 24 postoperative hours was measured was $6.72 \pm 5.7$ for gabapentin group and $24.13 \pm 47.01$ for control group, a statistically significant difference $(p<0.05)$; the prevalence of adverse effects was not statistically significant between groups.

Conclusion: These results indicate that gabapentin at doses of $600 \mathrm{mg}$ preoperative is a valid therapeutic option in preoperative analgesia for abdominal hysterectomy. Note that we have yet to elucidate the optimal effective dose and to present lower incidence of adverse effects.

Key words: Acute pain. Postoperative pain. Gabapentine. Abdominal hysterectomy. Patient controlled analgesia.

\section{RESUMEN}

Introducción: es bien conocido que el dolor es síntoma subjetivo y el dolor postoperatorio sigue teniendo preponderancia mundial, ya que a pesar de todos los avances aún no se ha podido erradicar por completo.

Objetivo: evaluar el efecto adyuvante en la intensidad del dolor agudo postoperatorio de $600 \mathrm{mg}$ de gabapentina administrada en el preoperatorio en pacientes sometidas a histerectomía abdominal en el Hospital Santo Tomás, de abril a agosto de 2014.

Métodos: se realizó un estudio de cohorte experimental prospectivo aleatorizado, controlado, doble ciego, en pacientes sometidas a histerectomía abdominal con anestesia general. Se aleatorizó en dos grupos: grupo gabapentina (gabapentina 600 mg 2 horas preoperatoria + analgesia con morfina a través de bomba de analgesia controlada por el paciente [PCA]) y grupo control (analgesia con morfina a través de bomba PCA; la dosis de morfina se calculó en función de rangos de peso a 0,016 $\mathrm{mg} / \mathrm{kg} / \mathrm{h}$ ). Se evaluó la intensidad del dolor con la escala verbal numérica (EVN) de 11 puntos a las 2, 6 y 24 horas, se cuantificó la cantidad de rescates administrados en el postoperatorio y se midió la prevalencia de efectos adversos en ambos grupos. 
Resultados: se reclutaron 72 pacientes, divididos en dos grupos homogéneos; la edad media fue de 44,72 $\pm 6,17$ años en el grupo gabapentina y 49,36 \pm 9,31 años en el grupo control. En cuanto al dolor postoperatorio, hubo diferencia estadísticamente significativa entre los dos grupos ( $<<0,05)$ : se encontró una diferencia en la EVN de 11 puntos, $4,27 \pm 2,32,1,94 \pm$ 1,95 y $0,77 \pm 1,28$ en el grupo gabapentina y $7,13 \pm 2,41$, $4,5 \pm 2,37$ y $3,25 \pm 2,18$ en el grupo control a las 2,6 y 24 horas respectivamente; se cuantificó la cantidad de rescates administrados por bomba de PCA durante las primeras 24 horas postoperatorias fue de 6,72 \pm 5,7 para el grupo gabapentina y 24,13 \pm 47,01 para el grupo control, una diferencia estadísticamente significativa $(\mathrm{p}<0,05)$; la prevalencia de efectos adversos no fue estadísticamente significativa entre ambos grupos.

Conclusión: estos resultados indican que la gabapentina en dosis de 600 mg preoperatoria es una opción terapéutica válida en analgesia preoperatoria para histerectomía abdominal. Cabe destacar que aún nos queda por dilucidar la dosis óptima efectiva y que presente menor incidencia de efectos adversos.

Palabras clave: Dolor agudo. Dolor postoperatorio. Gabapentina. Histerectomía abdominal. Analgesia controlada por el paciente.

\section{INTRODUCCIÓN}

El dolor agudo postoperatorio consiste en una de las principales complicaciones postquirúrgicas que afecta significativamente a la recuperación y reincorporación a las actividades diarias de cada paciente (1).

El dolor agudo cumple una función fisiológica de protección ante una lesión tisular con el fin de prevenir la lesión o disminuir los efectos de un mecanismo que, potencialmente, produzca mayor daño a una lesión existente.

El dolor agudo de origen postoperatorio se corresponde con el producido por una lesión tisular conocida y esperada que sobreviene a la intervención quirúrgica; no presenta utilidad diagnóstica o protectora, por lo que su tratamiento y prevención son prioritarios ante todo procedimiento quirúrgico (2).

El dolor conlleva respuestas fisiológicas y psicológicas en el paciente que pueden tener efectos deletéreos o no deseados. El dolor agudo es uno de los activadores del complejo de respuesta humoral y respuesta inmune; ante una lesión esta activación mantenida de forma prolongada altera los mecanismos de percepción del dolor, de tal manera que el dolor agudo y los sistemas inmunes de la lesión se correlacionan con efectos aditivos (1).

Clínicamente esta respuesta fisiológica, desencadenada por la lesión tisular y el dolor agudo, puede evidenciarse como inflamación, hiperalgesia, hiperglicemia, lipolisis, catabolismo proteico y cambios en el agua corporal e influjos de electrolitos, efectos cardiovasculares por activación del sistema simpático, trastornos de la coagulación y función inmune (3).
Además de los efectos fisiológicos mencionados, el dolor agudo también produce cambios psicológicos que incluyen ansiedad, insomnio, desmoralización, sentimientos de desesperanza y pérdida del control situacional, e incapacidad para comunicarse de forma efectiva. En pacientes ancianos se describe la aparición de disfunción cognitiva postoperatoria, aunque su etiología no es clara (2).

La gabapentina es un fármaco con estructura análoga al neurotransmisor GABA. Fue desarrollado en 1993 como adyuvante terapéutico en convulsiones parciales, con posterior apertura para distintos síndromes dolorosos crónicos. En 2002 es aprobado por la Food and Drug Administration (FDA) como agente terapéutico en neuralgia postherpética y en el Reino Unido se aprueba para todo tipo de dolor neuropático crónico (4). Posteriormente se propone su uso en el manejo del dolor agudo postquirúrgico con distintos esquemas de administración, pacientes y cirugías, obteniéndose resultados variados (4-9).

En el Hospital Santo Tomás se ha logrado reducir la incidencia del dolor agudo postoperatorio, pero aún distamos de lograr nuestro objetivo de un hospital sin dolor, siendo predecible la aparición del dolor postoperatorio.

Dentro de la gran variedad de cirugías que se realizan en este hospital encontramos la histerectomía abdominal, uno de los tipos de cirugía al que se asocia mayor incidencia de dolor agudo postoperatorio, de moderado a grave.

El objetivo del estudio es evaluar el efecto adyuvante de una dosis de $600 \mathrm{mg}$ de gabapentina preoperatoria en la intensidad del dolor agudo postoperatorio en pacientes sometidas a histerectomía abdominal.

\section{MÉTODOS}

Previo autorización del Comité de Ética e Investigación del Hospital Santo Tomás, realizamos un estudio de cohorte experimental prospectivo aleatorizado, controlado, doble ciego, en pacientes femeninas a las cuales se les realizó histerectomía abdominal con anestesia general, previo consentimiento informado de la paciente.

Se calculó la muestra utilizando la fórmula de población infinita y probabilidad de reducción del dolor según uso de opioides después de dosis única de gabapentina en cirugía mayor, siendo la muestra de 36 pacientes por grupo.

Se incluyeron pacientes femeninas mayores de 18 años, ASA I-II, llevadas a cirugía electiva. Se excluyó a pacientes alérgicas a gabapentinoides, morfina o AINE; pacientes con enfermedad renal o hepática, con diagnóstico de síndrome doloroso previo y antecedente de consumo de sustancias ilícitas.

El día previo al procedimiento quirúrgico se le realizó la evaluación preoperatoria y aleatorización a cada paciente por método de azar simple a través de sobres cerrados sin conocimiento del paciente y el anestesiólogo que realiza la 
evaluación del grupo asignado. El investigador principal, el cual recogió toda la muestra, realizó la evaluación del dolor y desconocía el grupo al cual pertenecía la paciente.

- Grupo de intervención o grupo gabapentina: se administran $600 \mathrm{mg}$ de gabapentina por vía oral 2 horas previas a la cirugía.

- Grupo control: no se administraran medicamentos adyuvantes u otra premedicación en el preoperatorio. Recibió una tableta de citrato de calcio de $1.500 \mathrm{mg}$ con forma farmaceútica similar a la gabapentina pero sin componente activo en analgesia.

La bomba de analgesia PCA se inició en el periodo postoperatorio inmediato. Las pacientes recibieron igual esquema de analgesia intravenosa compuesta por metamizol $50 \mathrm{mg} / \mathrm{kg}$ de peso, en bolos, morfina intravenosa por bomba de PCA a dosis de $0,016 \mathrm{mg} / \mathrm{kg} /$ hora en infusión continua y rescates a dosis de $0,00625 \mathrm{mg} / \mathrm{kg} / \mathrm{dosis}$, programados para un número máximo de 2 dosis por hora y tiempo de cierre entre dosis de 30 minutos. Se evaluó la intensidad de dolor en EVN de 11 puntos y se cuantificó la incidencia de náuseas, vómitos, prurito y sedación en escala de Ramsey, y se utilizó la escala de 0 a 100 para evaluar la satisfacción durante la marcha. Se realizaron evaluaciones a las 2, 6 y 24 horas postoperatorias.

El análisis estadístico se realizó con EpiInfo 7.1.4.0., utilizando medias, desviación estándar y t-de student para variables continuas, porcentajes y $\chi^{2}$ para variables cualitativas.

\section{RESULTADOS}

Se incluyeron 72 pacientes femeninas entre ASA I y ASA II distribuidas de forma homogénea en ambos grupos de investigación, 36 pacientes en cada grupo; la clasificación ASA se distribuyó así: grupo control 36,11\% y $63,89 \%$, y grupo gabapentina $30,56 \%$ y $69,44 \%$ para ASA I y ASA II, respectivamente. Las características demográficas fueron muy similares entre los grupos. Los diagnósticos más comunes fueron $80,5 \%, 8,33 \%$ y $1,11 \%$ en el grupo control y $86,11 \%, 11,11 \%$ y $2,78 \%$ en el grupo gabapentina para miomatosis uterina, prolapso genital y otros diagnósticos, donde se incluyen la displasia cervical y la hiperplasia endometrial, respectivamente para cada grupo. La edad media fue de 44,72 $\pm 6,17$ años en el grupo gabapentina y de 49,36 $\pm 9,31$ años en el grupo control. El peso promedio fue de 74,91 $\pm 16,67 \mathrm{~kg}$ para el grupo control y de 73,75 \pm $15,41 \mathrm{~kg}$ para el grupo gabapentina, lo cual nos lleva a un resultado no estadísticamente significativo.

La combinación de analgésicos (a elección del anestesiólogo a cargo del caso) más utilizada en el preoperatorio de las pacientes fue metamizol + tramadol en ambos grupos (Fig. 1).

En cuanto al dolor postoperatorio, hubo una diferencia estadísticamente significativa entre los dos grupos $(\mathrm{p}<0,05)$ : se encontró una diferencia en la EVN de 11 puntos, 4,27 \pm $2,32,1,94 \pm 1,95$ y $0,77 \pm 1,28$ en el grupo gabapentina y $7,13 \pm 2,41,4,5 \pm 2,37$ y $3,25 \pm 2,18$ en el grupo control a las 2,6 y 24 horas respectivamente, que corresponden a una reducción del 40-50\% en el grupo que recibió gabapentina preoperatoria con respecto al grupo control (Fig. 2).

Se cuantificó la cantidad de rescates administrados por la bomba de PCA durante las primeras 24 horas postoperatorias y estos fueron los hallazgos: 6,72 $\pm 5,7$ rescates para el grupo gabapentina y $24,13 \pm 47,01$ rescates para el grupo control, una diferencia estadísticamente significativa para una p 0.03 (Tabla I). El consumo de morfina en 24 horas para el grupo control fue de $23,24 \pm 2,97 \mathrm{mg} / 24 \mathrm{~h}$ y 20,42 $\pm 3,91 \mathrm{mg} / 24 \mathrm{~h}$ para el grupo gabapentina, dando como resultado p 0,01 y una reducción en el consumo de opioide de un 20\%, una diferencia estadísticamente significativa entre los grupos (Tabla II).

Evaluamos la incidencia de efectos adversos asociados al uso de analgésicos opioides y gabapentina durante el

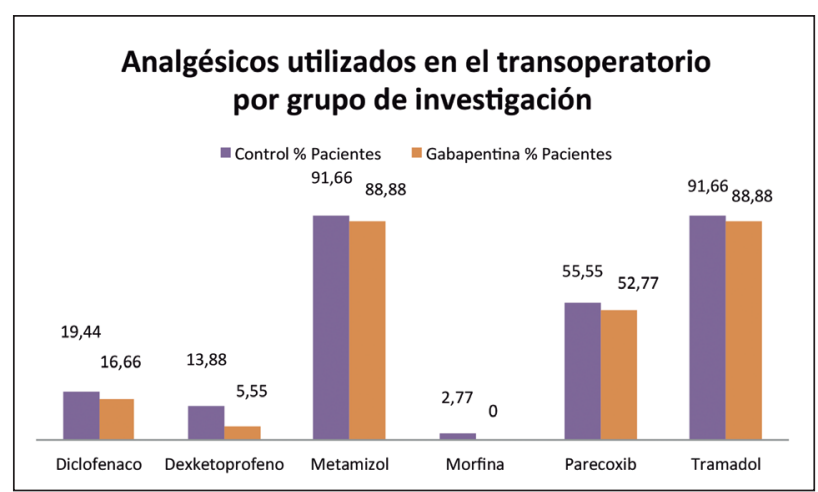

Fig. 1. Se presentan los analgésicos administrados durante el transoperatorio en porcentaje de pacientes que recibieron el fármaco por grupo.

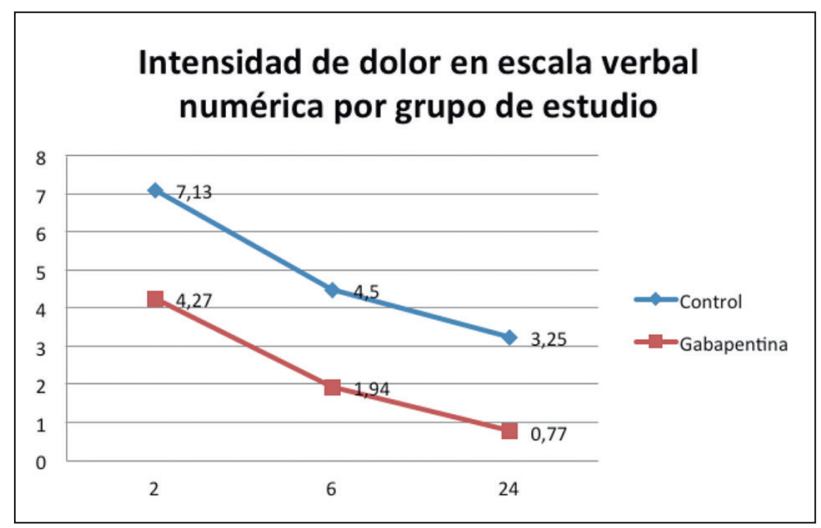

Fig. 2. Gráfica lineal de medias de intensidad de dolor en escala verbal numérica por tiempo. Resultados a las 2, 6 y 24 horas postoperatorias. 
postoperatorio y no se encontraron diferencias significativas entre ambos grupos (Tabla III). Esto demuestra que, en este caso, el uso de la gabapentina no aumenta o disminuye

TABLA I. RESCATES DE MORFINA SOLICITADOS EN 24 HORAS

\begin{tabular}{lcccc}
\hline & $n$ & Media & std & pValor \\
\hline Control & 36 & 24,13 & 47,01 & \\
Gabapentina & 36 & 6,72 & 5,7 & 0,03 \\
\hline
\end{tabular}

Media y desviación estándar de episodios de rescates analgésicos solicitados por bomba de analgesia controlada por el paciente.

Los valores de $\mathrm{p}>0,05$ no se consideran significativos.

TABLA II. CONSUMO TOTAL DE MORFINA EN 24 HORAS

\begin{tabular}{lccccc}
\hline & $n$ & Media & std & tValor & $p$ Valor \\
\hline Control & 36 & 23,24 & 2,97 & & \\
Gabapentina & 36 & 20,42 & 3,91 & & 0,01
\end{tabular}

Media y desviación estándar de miligramos de morfina administrados por bomba de analgesia controlada por el paciente. Los valores de $\mathrm{p}>0,05$ no se consideran significativos. el riesgo de presentar efectos adversos, como lo expuesto en distintas revisiones en diferentes grupos de pacientes y procedimientos.

Evaluamos la satisfacción durante el inicio de la marcha. Se estableció una escala subjetiva de 0 a 100 para la evaluación de este acápite, siendo $0 \%$ el dolor no permite la bipedestación o la marcha y $100 \%$ que deambula y realiza sus actividades sin limitación por dolor; así, el grupo control tuvo una satisfacción del 67,77 \pm $25,31 \%$ frente al grupo gabapentina, que manifestó un $93,11 \pm 10,61 \%$ de satisfacción, dando como resultado final una diferencia estadísticamente significativa con $\mathrm{p}$ $<0,01$ (Tabla IV).

TABLA IV. SATISFACCIÓN AL INICIAR LA MARCHA

\begin{tabular}{lcccc}
\hline & Media & std & tValor & $p$ Valor \\
\hline Control & 67,77 & 25,31 & & \\
Gabapentina & 93,11 & 10,61 & $-5,55$ & 0,00 \\
\hline
\end{tabular}

Media y desviación estándar. Se realiza prueba t-student para evaluar significancia estadística. Los valores $\mathrm{p}>0,05$ no son significativos.

TABLA III. INCIDENCIA DE EFECTOS ADVERSOS EN POSTOPERATORIO

\begin{tabular}{|c|c|c|c|c|c|c|c|}
\hline & & Control & Gabapentina & $O R$ & $\chi^{2}$ & IC & pValue* \\
\hline \multirow{3}{*}{ Náuseas } & 2 horas & 27,77 & 11,11 & 3,07 & 3,14 & $0,86-10,95$ & 0,076 \\
\hline & 6 horas & 44,44 & 27,77 & 2,07 & 2,13 & $0,77-5,55$ & 0,140 \\
\hline & 24 horas & 58,33 & 36,11 & 2,47 & 3,15 & $0,95-6,40$ & 0,060 \\
\hline \multirow{3}{*}{ Vómitos } & 2 horas & 2,77 & 2,77 & 1,00 & 0,51 & $0,06-16,62$ & 0,470 \\
\hline & 6 horas & 5,55 & 5,55 & 1,00 & 0,26 & $0,13-7,51$ & 0,600 \\
\hline & 24 horas & 19,44 & 13,88 & 1,49 & 0,39 & $0,42-5,24$ & 0,520 \\
\hline \multirow{3}{*}{ Prurito } & 2 horas & 0,00 & 2,77 & $\mathrm{NC}$ & 1,00 & $\mathrm{NC}$ & 0,310 \\
\hline & 6 horas & 0,00 & 2,77 & $\mathrm{NC}$ & 1,00 & $\mathrm{NC}$ & 0,310 \\
\hline & 24 horas & 0,00 & 5,55 & $\mathrm{NC}$ & 2,02 & $\mathrm{NC}$ & 0,150 \\
\hline \multirow{3}{*}{ R. urinaria } & 2 horas & 0,00 & 0,00 & $\mathrm{NC}$ & $\mathrm{NC}$ & $\mathrm{NC}$ & 1,00 \\
\hline & 6 horas & 0,00 & 0,00 & $\mathrm{NC}$ & $\mathrm{NC}$ & $\mathrm{NC}$ & 1,00 \\
\hline & 24 horas & 2,77 & 0,00 & $\mathrm{NC}$ & 1,00 & $\mathrm{NC}$ & 0,310 \\
\hline \multirow{3}{*}{ Sedación } & 2 horas & 38,88 & 36,11 & NA & 0,58 & NA & 0,800 \\
\hline & 6 horas & 5,55 & 13,88 & NA & 1,4 & NA & 0,230 \\
\hline & 24 horas & 5,55 & 0,00 & NA & 2,02 & $\mathrm{NA}$ & 0,150 \\
\hline
\end{tabular}

Valores de incidencia de efectos adversos por grupo de estudio presentado en porcentajes. *Valores $\mathrm{p}>0,05$ no se consideraron significativos. **Sedación evaluada en escala de Ramsey, se consideró positivo puntuación 3 o mayor. Puntuación máxima observada en los grupos: Ramsey 3. 


\section{DISCUSIÓN}

A pesar de tener una muestra pequeña hemos visto diferencias estadísticamente significativas importantes para los objetivos de este estudio en particular.

Nuestros resultados no distan de los resultados obtenidos en otros estudios, como los de Turan 2004 (10), donde se utilizaron $1.200 \mathrm{mg}$ de gabapentina oral preoperatoria, al igual que en el 2006 (11) y Frouzanfard en 2013 (12), mostrando reducción de los valores de dolor agudo postoperatorio y una reducción del consumo de opioides, tal como reportan Freouzanfard y cols. (12), después de recibir gabapentina preoperatoria en histerectomía abdominal.

La incidencia de efectos adversos, a pesar de estar presentes, no tiene valor estadísticamente significativo; esto pudiera ser debido al tamaño de la muestra, pero en este estudio es consistente con estudios como el de Durmus y cols. (13), que reporta que las náuseas y vómitos son menos comunes en el grupo gabapentina que en el grupo control.

Estos resultados indican que la gabapentina en dosis de $600 \mathrm{mg}$ preoperatoria es una opción terapéutica válida en analgesia preventiva para histerectomía abdominal. Cabe destacar que aún queda por dilucidar la dosis óptima efectiva que presente buena tolerancia, menor incidencia de efectos adversos y buen manejo del dolor postoperatorio.

\section{Limitaciones}

Las posibles debilidades de este estudio estriban en la cantidad de pacientes totales y en la falta de uniformidad en la analgesia transoperatoria, lo cual se podría mejorar en próximos estudios.

\section{AGRADECIMIENTOS}

Al Servicio de Ginecología del Departamento de Ginecología y Obstetricia del Hospital Santo Tomás por permitirnos manejar la analgesia de las pacientes de manera exclusiva durante las primeras 24 horas.

\section{CORRESPONDENCIA:}

Marjorie Errigo Pinillo

Servicio de Anestesiología

Departamento de Áreas Críticas

Hospital Santo Tomás. Panamá

e-mail: marerrigomd@gmail.com

\section{BIBLIOGRAFÍA}

1. Kehlet H, Dahl JB. Anaesthesia, surgery, and challenges in postoperative recovery. Lancet 2003;362(9399):1921-8.

2. Liu SS, Block BM, Wu CL. Effects of perioperative central neuraxial analgesia on outcome after coronary artery bypass surgery: A meta-analysis. Anesthesiology 2004;101(1):153-61.

3. Cross, Shelley A. MD. Pathophysiology of pain. Mayo Clinic Proc 1994;69:375-83.

4. Kong V, Irwin M. Gabapentin: A multimodal perioperative drug? Br J Anaesth 2007;99:775-86.

5. Ho K, Tang Y, Habib G. Gabapentin and postoperative pain - A systematic review of randomized control trials. Pain 2006;126:91-101.

6. Clarke H, Kirkham K, Orser B. Gabapentin reduces preoperative anxiety and pain catastrophizing in highly anxious patients prior to major surgery: A blinded randomized placebo-controlled trial. Can J Anesth/J Can Anesth 2013;60:432-43.

7. Seib R, Paul J. Preoperative gabapentin for postoperative analgesia: A meta-analysis. Can J Anesth 2006;53(5):461-9.

8. Tippana E, Hanuman K. Do surgical patients benefit from perioperative gabapentin/pregabalin? A Systematic Review of Efficacy and Safety. Anesth Analg 2007;104(6):1545-56.

9. Mathiesen O, Moiniche S, Dahl J. Gabapentin in postoperative pain: A quality and quantity systematic review, with focus on procedure. BMC Anesthesiology 2007;7:6.

10. Turan A, Karamanlioğlu B, Memiş D, Usar P, Pamukçu $\mathrm{Z}$, Türe M. The analgesic effects of gabapentin after total abdominal hysterectomy. Anesth Analg 2004;98(5):1370-3.

11. Turan A, White PF, Karamanlioglu B, Memis D, Tas , dogan M, Pamukcu Z, et al. Gabapentin: An alternative to the cyclooxygenase- 2 inhibitors for perioperative pain management. Anesth Analg 2006;102:175-81.

12. Frouzanfard F, Fazel MR, Abolhasani A, Fakharian E, Mousavi G, Moravveji, A. Effects of gabapentin on pain and opioid consumption after abdominal hysterectomy. Pain Research \& Management: The Journal of the Canadian Pain Society 2013;18(2):94-6.

13. Durmus M, Kadir But A, Saricicek V, Ilksen Toprak H, Ozcan Ersoy M. The post-operative analgesic effects of a combination of gabapentin and paracetamol in patients undergoing abdominal hysterectomy: A randomized clinical trial. Acta Anaesthesiol Scand 2007;51(3):299-304. 